\title{
Temperament and Character Dimensions: Correlates of Impulsivity in Morphine Addicts
}

\author{
Moslem Abassi," and Abbas Abolghasemi ${ }^{2}$ \\ ${ }^{1}$ Department of Psychology, Faculty of Literature and Human Sciences, Salman Farsi University of Kazerun, Kazerun, IR Iran \\ ${ }^{2}$ Department of Psychology, Faculty of Humanities, University of Gilan, Rasht, IR Iran \\ ${ }^{*}$ Corresponding author: Moslem Abassi, Department of Psychology, Faculty of Literature and Human Sciences, Salman Farsi University of Kazerun, Kazerun, IR Iran. Tel: \\ +98-7212214425, E-mail: abbasi@kazeronsfu.ac.ir
}

Received 2014 June 13; Revised 2014 November 4; Accepted 2014 November 17.

\begin{abstract}
Background: Given the role of temperament and character dimensions on impulsivity in addicts, the purpose of this study was to temperament and character dimensions: correlates of impulsivity in morphine addicts.

Objectives: The aim of this study was to determine and verify the association of temperament and character dimensions with impulsivity in morphine addicts.

Patients and Methods: The research method was descriptive and correlational. The study sample consisted of 120 morphine addicts referred to drug addiction treatment centers in Ardabil city in 2013. The participants were selected through convenience sampling method from 5 centers. We used impulsivity scale as well as temperament and character inventory to collect data.

Results: The results showed that significant relationship existed between impulsivity and characteristics such as novelty seeking, harm avoidance, reward dependence, persistence, self-directedness, and cooperativeness, while no significant relationship between impulsivity and self-transcendence was observed. The results of the multiple regression analysis showed that $47 \%$ of the impulsivity variance was explained by temperament and character dimensions.

Conclusions: These findings suggest that temperament and character dimensions are associated with impulsivity. The findings also have important implications for prevention, pathology, and treatment in the morphine addicts.
\end{abstract}

Keywords: Behavior, Addictive, Character, Morphine, Temperament

\section{Background}

Individuals with morphine addicts are more prone to act impulsivity. Also morphine addicts have a close relation to this impulsivity. Then we explored the role of them to predict high risk behaviors in impulsivity morphine addicts.

According to official statistics, there are 1.2 to 2 million drug addicts in the country with an average age of 18 years old, and 11 millions are entangled in the problem of their own or relatives' addiction (1). Various causes have been accounted for substance abuse. For example, some people seek to be accepted by the community and try to make people believe they are already grown-ups. The society and companions reciprocally play roles in increasing substance use. In some social groups, substance use is a very common condition of being accepted by group members. This claim is based on a body of research proving that offers by friends to use substance leads to increased use $(2,3)$. Substance abuse habits are also dependent on psychological variables, including behavioral and personality traits (4).

On the other hand, now there is an agreement that impulsivity has a major role in the development of substance use. Impulsivity is basically involved in both the onset and development of adolescent substance abuse. Barratt showed in a study that psychopaths, especially those with problems related to substance abuse, are more prone to impulsive behaviors (5). Young suggests that substance abuse makes it difficult to control impulsivity. Through separate studies on cocaine-dependent individuals, Moeller et al. concluded that impulsivity is an important predictor of cocaine use (6). Dawe and Loxton concluded in a study that impulsivity has an important role in the prediction and development of adolescent substance abuse (7). Coskunpinar suggests that there is a small and significant effect size between impulsivity and substance-related attentional bias, which was moderated by impulsivity measurement type. The relationship between behavioral impulsivity and substance-related attentional bias was stronger than trait impulsivity and substance-related attentional bias. Different components of behavioral impulsivity and trait impulsivity did not affect this relationship $(8,9)$.

One of the variables which may play a role in drug addiction is temperament and character dimensions. Cloninger's theory about temperament and character dimensions, especially with regard to substance abuse

Copyright (c) 2015, Zahedan University of Medical Sciences. This is an open-access article distributed under the terms of the Creative Commons Attribution-NonCommercial 4.0 International License (http://creativecommons.org/licenses/by-nc/4.0/) which permits copy and redistribute the material just in noncommercial usages, provided the original work is properly cited. 
Abassi M et al.

is important for a number of reasons. The first is that behavior assessment in temperament and character dimensions (e.g. impulsivity, intolerance, etc.) is an appropriate idea for the onset and continuation of drug dependence (10). For example, maladaptive impulsivity and cue reactivity predict relapse in cocaine addiction. Also impulsive people with high novelty seeking trait will be more frustrated when being deprived of drug reward, and consequently may be affected by more negative experiences. The second is to assess genetic background of temperament and character traits, which may play the mediating role in the association between genotypes and substance use behavior (11). For example, Elovainio et al. demonstrated that novelty seeking indirectly affects substance use behavior and by the type 4 dopamine receptor (D4). Third, systems involved in processing and responding to inclination, aversion, and new stimuli are associated with temperament dimensions that may lay the groundwork for substance use behavior. Fourth, pharmacological association with temperament and character dimensions may relate to the pathophysiology of drug addiction (12).

Some studies reported that harm avoidance and reward dependence are partially related to the onset of smoking and its dependence severity (13). However, other studies reported negative relationship between reward dependence and drug dependence Gerra et al. (14). showed through a study that novelty seeking had negative relationship with performance of dopaminergic system in two groups of addicts and normal but the relationship with serotonergic system was negative in addicts group. Moreover, the research outcomes showed that harm avoidance has positive relationship with serotonergic system in normal group; however, that relationship was not significant in addicts. Leventhal et al. (15) demonstrated that individuals with high levels of novelty seeking report more nicotine withdrawal symptoms, negative impacts, and craving to smoke, while withdrawing and abstaining from drug use. However, no relationship was observed between reward dependence dimension and smoking abstinence in that research. Evren et al. (16) demonstrated that among temperament dimensions, drug-dependent patients gained higher scores in novelty seeking and lower scores in reward dependence compared to alcohol-dependent patients. Drug-dependent patients had lower scores in self-directedness and persistence compared to temperament dimensions.

On the whole, considering the role of impulsivity in development of various symptoms of psychiatric disorders, and existence of contradictory findings and limited research in this field, administration of this study seems to be necessary. Also, because impulsivity is only associated with substance use, it is critical to delineate its role on substance use co-occurrence. Therefore, the purpose of the present research was to determine the association of temperament and character dimensions with impulsivity in morphine addicts.

\section{Objectives}

The aim of this study was to determine and verify the association between temperament and character dimensions as well as impulsivity in morphine addicts.

\section{Patients and Methods}

This research was descriptive and correlational. Impulsivity is predicted through temperament and character dimensions. The statistical population for this research included all outpatient morphine addicts referred at drug addiction treatment centers of Ardabil city in 2013. The research sample consisted of 120 morphine addicts selected from outpatients referred at drug addiction treatment centers of Ardabil. First, 5 centers were randomly selected out of all addiction treatment centers there. Then, 120 addicts were selected out of all admitted morphine addicts via convenience sampling. The minimum sample size recommended for causal-comparative studies is at least 30 persons for each group. In the present study, 60 subjects were selected for each group in order to increase the validity of the research.

The inclusion criteria for this study were as follow:

1- Morphine addiction,

2- Age range of 20 - 40 years,

3- Education range from high school diploma to bachelor's degree,

4- No chronic physical illness,

5- No psychosis, and

6- Being addicted for at least 2 years.

Instruments utilized for data collection were as presented in following sections.

\subsection{Impulsivity Scale}

Impulsivity Scale was developed by Barratt (5). It has 30 items to which the subjects should answer on a 4-point scale (never, frequently, often, and almost always). This scale measures three components; lack of planning, kinesthetic, and cognitive impulsivity. As Abolghasemi, Poor Kord, and Narimani reported, the Cronbach $\alpha$ coefficient and retest reliability coefficient (after one month) for this scale were 0.87 and 0.79 , respectively.

\subsection{The Temperament and Character Inventory}

Cloninger et al. (17) developed this inventory to measure temperament, biogenetics, and acquired character. It consisted of 125 items to which each subject should answer as true or false. According to this inventory, temperament has 4 dimensions (novelty seeking, harm avoidance, reward dependence, and persistence) and character has 3 dimensions (self-directedness, cooperativeness, and selftranscendence). Its retest reliability coefficient obtained by Kaviani and Stillwell (18) in 7 scales ranged from 0.61 to 0.96 . No significant correlation was found between the 4 scales of temperament and the 3 scales of character. This means that the scales are independent. Coefficient of cor- 
relation between short form of the inventory and its long from was 0.62 .

\subsection{Procedure}

After receiving letter of introduction from education department for referring to outpatient drug addiction treatment centers, we identified those exposed to addiction that were diagnosed as morphine addicts. At the next stage, subjects were administered the Impulsivity Scale and Temperament and Character Inventory. Then the collected data were analyzed through Pearson's correlation coefficient and multiple regression analyses using the SPSS software.

\section{Results}

The frequency of educational degrees of the participants were as follows: $20 \%$ with middle level certificate, 42.5\% with high school diploma, $25 \%$ with associate degree, and $12.5 \%$ with a bachelor's degree and higher. The mean (standard deviation) age of addicts was 36.45 (4.37) year, with a range of 20 - 40 years old. About $6.7 \%$ were divorced, $51.7 \%$ single, and $47.7 \%$ married. Also about $5 \%$ were jailed previously and finally, $59.2 \%$ were unemployed.

Table 1 demonstrates the mean and standard deviation scores of temperament and character dimensions and impulsivity in drug addicted patients.
Results of Pearson correlation coefficient showed that novelty seeking $(r=0.54)$, harm avoidance $(r=0.33)$, reward dependence $(r=-0.44)$, persistence $(r=-0.41)$, selfdirectedness $(r=-0.57)$, and cooperativeness $(r=-0.52)$ are significantly correlated with impulsivity $(\mathrm{P}<0.001)$, but no significant relationship was found between selftranscendence $(r=-0.46)$ and impulsivity.

Table 1. Mean and Standard Deviation Scores for Temperament and Character Dimensions and Impulsivity in Drug Addicted Patients

\begin{tabular}{lc}
\hline Variable & Mean \pm SD \\
\hline Novelty Seeking & $10.26 \pm 3.09$ \\
\hline Persistence & $2.80 \pm 1.38$ \\
\hline Harm Avoidance & $10.16 \pm 3.28$ \\
\hline Reward Dependence & $11.64 \pm 3.15$ \\
\hline Self-directedness & $8.78 \pm 3.68$ \\
\hline Cooperativeness & $14.69 \pm 4.20$ \\
Self-transcendence & $10.42 \pm 2.85$ \\
\hline Cognitive & $19.53 \pm 4.38$ \\
\hline Motor & $25.32 \pm 7.28$ \\
\hline Plan & $27.76 \pm 6.57$ \\
\hline Total & $72.60 \pm 15.48$ \\
\hline
\end{tabular}

Table 2. Coefficients of Correlation Between Temperament and Character Dimensions and Impulsivity

\begin{tabular}{|c|c|c|c|c|}
\hline Variable & Cognitive & Motor & Plan & Total \\
\hline Novelty Seeking & $0.44^{\mathrm{a}}$ & $0.45^{\mathrm{a}}$ & $0.48^{\mathrm{a}}$ & $0.54^{\mathrm{a}}$ \\
\hline Harm Avoidance & $0.32^{\mathrm{a}}$ & $0.24^{\mathrm{b}}$ & $0.30^{\mathrm{a}}$ & $0.33^{\mathrm{a}}$ \\
\hline Reward Dependence & $-0.36^{\mathrm{a}}$ & $-0.39^{\mathrm{a}}$ & $-0.36^{\mathrm{a}}$ & $-0.44^{\mathrm{a}}$ \\
\hline Persistence & $-0.28^{C}$ & $-0.38^{\mathrm{a}}$ & $-0.37^{\mathrm{a}}$ & $-0.41^{\mathrm{a}}$ \\
\hline Self-directedness & $-0.48^{\mathrm{a}}$ & $-0.48^{\mathrm{a}}$ & $-0.49^{\mathrm{a}}$ & $-0.57^{\mathrm{a}}$ \\
\hline Cooperativeness & $-0.43^{a}$ & $-0.43^{\mathrm{a}}$ & $-0.46^{\mathrm{a}}$ & $-0.52^{\mathrm{a}}$ \\
\hline Self-transcendence & -0.04 & 0.06 & 0.04 & 0.03 \\
\hline
\end{tabular}

$\mathrm{a}_{\mathrm{P}}<0.001$

$\mathrm{b}_{\mathrm{P}}<0.05$

${ }^{\mathrm{C}} \mathrm{P}<0.01$.

Table 3. Summary of the Results of Multivariate Regression Analysis for the Prediction of Impulsivity Through Temperament and Character Dimensions ${ }^{\mathrm{a}}$

\begin{tabular}{|c|c|c|c|c|}
\hline \multirow[t]{2}{*}{ Variable } & \multirow{2}{*}{$\frac{\text { Standardization }}{\beta}$} & \multicolumn{2}{|c|}{ Un Standardization } & \multirow[t]{2}{*}{$\mathbf{t}$} \\
\hline & & SE & $\mathbf{b}$ & \\
\hline Constant & - & 9.69 & 87.36 & $9.01^{b}$ \\
\hline Novelty Seeking & 0.539 & 0.390 & 2.703 & $6.96^{\mathrm{b}}$ \\
\hline Cooperativeness & -0.349 & 0.295 & -1.288 & $-4.36^{\mathrm{C}}$ \\
\hline Self-directedness & -0.259 & 0.349 & -0.910 & $2.65^{\mathrm{d}}$ \\
\hline Persistence & -0.203 & 0.859 & -2.273 & $2.61^{d}$ \\
\hline
\end{tabular}

\footnotetext{
${ }_{\mathrm{a}} \mathrm{R}^{2}=469, \mathrm{~F}=20.10, \mathrm{P}<0.001$.

$\mathrm{b}_{\mathrm{P}<0.001}$

$\mathrm{c}_{\mathrm{P}<0.01 \text {. }}$

$\mathrm{d}_{\mathrm{P}}<0.05$.
} 
Abassi M et al.

To determine the impact of temperament and character dimensions as predictor variables and impulsivity as the criterion variable, multivariate regression analysis was used. As it is seen in Table 3, results show that about $47 \%$ of the variance in impulsivity is explained by temperament and character dimensions. Considering beta values, dimensions of novelty seeking ( $\beta=0.539)$, cooperativeness ( $\beta=-0.349)$, self-directedness $(\beta=-0.259)$, and persistence ( $\beta=-0.203)$ are respectively the strongest variables for predicting impulsivity in morphine addicts.

\section{Discussion}

The purpose of the present research was to determine the association of temperament and character dimensions with impulsivity in morphine addicts. Research results showed that there was a significant and positive relationship between novelty seeking and impulsivity. In other words, individuals with higher levels of novelty seeking appear to be more impulsive and more inclined to use negative emotion regulation strategies and, consequently use narcotics. This finding is consistent with the results of other studies $(3,5,19)$, indicating the association of novelty seeking with impulsivity in those who are addicted to narcotic drugs. The evidence indicates that drug addicts have higher degrees of novelty seeking. Temperament of those with drug addiction is unstable due to high novelty seeking (impulsive, rapidly reactive), high harm avoidance (anxious and shy), low reward dependence (cool and recluse), and persistence. As a result, they usually experience dysthymia with a combination of anxiety and anger, and handle their social problems and strong feelings with primitive ways.

The results showed that there was a significant and positive relationship between harm avoidance and impulsivity. In other words, higher harm avoidance people are more impulsive as well. This finding is consistent with results of other studies $(8,9)$, based on the association of harm avoidance with impulsivity in narcotic drugs addicts. Results indicated that individuals with narcotic drugs addiction are much affected by harm avoidance (anxiety and shyness). Thus, high harm avoidance (identified with characteristics like escaping and avoiding from risky situations, caution, and seclusion) functions as a barrier to addiction and irritability symptoms. Such persons develop an unreal self-esteem and act without considering its harmful situational and behavioral consequences. Having low levels of harm avoidance characteristics like self-confidence versus uncertainty, leads to the individual's lots of activities with minimum discomfort. Harms of this state are lack of responsiveness to hazard and unrealistic optimism. And its potential hazardous outcomes are in situations with high risk probability and totally in direction of increase the intensity of symptoms such as irritability and impulsivity of those with drug addiction (8). To explain this finding, those with higher scores on novelty seeking and harm avoidance are not able to control themselves and consequently will probably have more tendency to use narcotics. These people are impulsive, nervous, anxious, timid, uncertain, hopeless, passive, negativist, pessimist, and insecure.

The results also showed that there was a significant and negative relationship between reward dependence and impulsivity in morphine addicts. In other words, individuals with morphine addiction with lower reward dependence are more impulsive. This finding is consistent with the results of other researches indicating the negative relationship between reward dependence and impulsivity in narcotics addicts $(9,10)$. Results showed that the less persistent the narcotics addicted people were, the more impulsive they would be. Impulsivity can be explained as a preference for immediate rewards, inclination to adventurism, novelty seeking, finding simple ways of gaining access to rewards, lack of persistence and insistence in accomplishing things, and also short personal reaction time (16). Furthermore, self-directedness and cooperativeness have significant and negative relationship with impulsivity in morphine addicts. In other words, morphine addicts with lower self-directedness and cooperativeness are more impulsive. This finding is consistent with the results gained from other researches indicating negative relationship of self-directedness and cooperativeness with impulsivity in drug addicts (15). Results show that individuals with narcotic drugs addiction display lower levels of self-directedness and cooperativeness. To explain this finding, it can be said that low levels of self-directedness and cooperativeness in such people are indicative of avoidance concept and response inhibition based on neurological approaches. This is an issue that causes drug addicts to be more exposed to the risk of impulsivity and behavioral problems $(10,11)$. Low levels of self-directedness and cooperativeness are associated with high levels of behavioral problems, impulsivity, anxiety, depression, attention problems, and violence in drug addicts. Moreover, individuals with low scores on reward dependence and self-directedness have cool, recluse, easily hurt, and irresponsible behavior. Those with low levels of cooperativeness are self-conceited, prejudiced, reproached, and vengeful. Considering these traits, it is completely rational that addicted individuals gain high scores on novelty seeking and harm avoidance and low scores on reward dependence, persistence, selfdirectedness, and cooperativeness. Results also showed that there was no significant relationship between selftranscendence and impulsivity. This finding is inconsistent with the results from other researches, indicating negative relationship of self-transcendence with impulsivity in narcotics drugs addicts (16). It might be due to the fact that self-transcendence accompanies religious faith and self-possession as well as unconditional patience, and together they act as a hindrance to impulsivity in these persons. 
Abassi M et al.

Results of multivariate regression analysis showed that temperament and character dimensions predict 70\% of the variance in impulsivity of morphine addicts. Dimensions of novelty seeking, cooperativeness, self-directedness, and persistence are the strongest variables for predicting impulsivity of morphine addicts. This result proves that $30 \%$ of the variance are effective on response inhibition and the rest of it by factors explained by other variables (e.g. personality, cognitive, and motivational variables). Moreover, considering no corresponding findings in the literature, it can be inferred from this finding that dimensions of novelty seeking, cooperativeness, selfdirectedness, and persistence have significant impact on impulsivity in morphine addicts. Therefore, more accuracy is needed to be applied in further research in order to clear this issue.

Regarding novelty seeking as the strongest predictor variable for impulsivity, it can be claimed that those with novelty seeking usually have a tendency toward exploratory activities, are interested in stimulation and excitement, are against monotony, and tend to experience new things. Besides, these persons take decisions with little information and hastiness; the traits that cause them to be exposed to more risks and eventually resort to narcotic drugs.

In conclusion, our data emphasize the association of temperament and character dimensions with impulsivity. Better understanding of these dimensions could help to improve addicts' behaviors and reduce the risk of relapses and may have a direct impact on psycho-educative interventions.

These observations need to be replicated in a larger population; further studies are also necessary to understand the biological aspects of personality that contribute to the relapses and severity of addiction. Lack of enough trust in responses of addicted people; unisex of the sample (men); confinement to one city (Ardabil); and no control over the kind of narcotics, consumption rate and drugs are the limitations of this research that should be considered in generalizing the results. More reliable results can be gained by studying individuals who has not started using methadone yet.

We also suggest that similar researches be done on female narcotic drugs addicts and addicts of different ages too. Finally, morphine addicts could be trained to learn self-control and emotion-regulation skills in the form of treatment projects.

\section{Acknowledgments}

We thank all people who participated in this study.

\section{Footnote}

Authors' Contribution:Moslem Abassi designed the study and collected the data. Abbas Abolghasemi wrote the abstract of the article and revised the article.

\section{References}

1. Shafiei M, Rahgozar A, Rahgozar M. The effect of education on knowledge, attitude and practice of family addicts.; Addiction the third congress.; Yazd. 2004. pp. 43-35.

2. Sohrabi F. Fundamental of spiritual intelligence. J Fund Health. 1987;1(1):30-3.

3. Dibajniya P, Bakhtiyari M. Status of mental health in students of Shahid Beheshti University. J Ardabil Univ. 2001;1(4):27-31.

4. Finn PR, Hall J. Cognitive ability and risk for alcoholism: shortterm memory capacity and intelligence moderate personality risk for alcohol problems. J Abnorm Psychol. 2004;113(4):569-81. doi:10.1037/0021-843X.113.4.569. [PubMed:15535789]

5. Barratt ES. Impulsivity: Integrating cognitive, behavioral, biological, and environmental data. Washington, DC: American Psychological Association; 1993.

6. Moeller FG. Increased impulsivity in cocaine dependent subjects independent of antisocial personality disorder and aggression. Drug Alcohol Dependence. 2002;68(1):105-11. [PubMed:12167556]

7. Da S, Loxton NJ. The role of impulsivity in the development of substance use and eating disorders. Neuroscience and Behavioral Reviews. 2004;28(3):343-51. doi:10.1016/j.neubiorev.2004.03.007.

8. Coskunpinar A, Cyders MA. Impulsivity and substance-related attentional bias: a meta-analytic review. Drug Alcohol Depend. 2013;133(1):1-14. doi: 10.1016/j.drugalcdep.2013.05.008. [PubMed: 23746428]

9. Wallace IC, Popp E, Mondore S. Safety climate as a mediator between foundation climates and occupational accidents: a group-level investigation. J Appl Psychol. 2006;91(3):681-8. doi: 10.1037/0021-9010.91.3.681. [PubMed:16737363]

10. Cloninger CR. Brain networks underlying personality development. New York: Raven Press.; 2008. pp. 183-20.

11. Cunningham KA, Anastasio NC. Serotonin at the nexus of impulsivity and cue reactivity in cocaine addiction. Neuropharmacology. 2014;76 Pt B:460-78. doi: 10.1016/j.neuropharm.2013.06.030. [PubMed: 23850573]

12. Elovainio M, Kivimäki M, Viikari J, Ekelund J, Keltikangas-Järvinen L. The mediating role of novelty seeking in the association between the type 4 dopamine receptor gene polymorphism and cigarette-smoking behavior. Personality and Individual Differences. 2005;38(3):639-645. doi:10.1016/j.paid.2004.05.018.

13. Flagel SB, Waselus M, Clinton SM, Watson SJ,Akil H. Antecedents and consequences of drug abuse in rats selectively bred for high and low response to novelty. Neuropharmacology. 2014;76 Pt B:425-36. doi:10.1016/j.neuropharm.2013.04.033. [PubMed:23639434]

14. Gerra G, Zaimovic A, Timpano M, Zambelli U, Begarani M, and et al. Neuroendocrine correlates of temperament traits in abstinent opiate addicts. J Subst Abuse. 2000;11(4):337-45. [PubMed:11147231]

15. Leventhal AM, Waters AJ, Boyd S, Moolchan ET, Heishman SJ, Lerman C, et al. Associations between Cloninger's temperament dimensions and acute tobacco withdrawal. Addict Behav. 2007;32(12):2976-89. doi:10.1016/j.addbeh.2007.06.014. [PubMed: 17624682]

16. Evren C, Evren B, Yancar C, Erkiran M. Temperament and character model of personality profile of alcohol- and drug-dependent inpatients. Compr Psychiatry. 2007;48(3):283-8. doi: 10.1016/j. comppsych.2006.11.003. [PubMed:17445524]

17. Cloninger CR, Przybeck TR, Svrakic DM, Wetzel R. The temperament and character inventory (TCI): A guide to its development and use. USA: Center for Psychobiology of Personality; 1994. pp. 23-175

18. Kaviani N, Stillwell Y. An evaluative study of clinical preceptorship. Nurse Educ Today. 2000;20(3):218-26. doi: 10.1054/ nedt.1999.0386. [PubMed:10820576]

19. Kumpfer KL. Factors and processes contributing to resilience. Resilience and development. New York: Springer; 2002.pp. 179-224. 